\title{
Multi-orbital-phase and Multiband Characterization of Exoplanetary Atmospheres with Reflected Light Spectra
}

\author{
Mario Damiano ${ }^{1}$ (D), Renyu $\mathrm{Hu}^{1,2}$ (1), and Sergi R. Hildebrandt ${ }^{1,3}$ (10) \\ ${ }^{1}$ Jet Propulsion Laboratory, California Institute of Technology, Pasadena, CA 91109, USA; mario.damiano@jpl.nasa.gov \\ ${ }^{2}$ Division of Geological and Planetary Sciences, California Institute of Technology, Pasadena, CA 91125, USA \\ ${ }^{3}$ Division of Physics, Mathematics and Astronomy, California Institute of Technology, Pasadena, CA 91125, USA \\ Received 2020 July 7; revised 2020 September 3; accepted 2020 September 8; published 2020 October 15
}

\begin{abstract}
Direct imaging of widely separated exoplanets from space will obtain their reflected light spectra and measure atmospheric properties. Previous calculations have shown that a change in the orbital phase would cause a spectral signal, but whether this signal may be used to characterize the atmosphere has not been shown. We simulate starshade-enabled observations of the planet $47 \mathrm{UMa} b$, using the present most realistic simulator Starshade Imaging Simulation Toolkit for Exoplanet Reconnaissance to estimate the uncertainties due to residual starlight, solar glint, and exozodiacal light. We then use the Bayesian retrieval algorithm EXOREL ${ }^{\mathfrak{R}}$ to determine the constraints on the atmospheric properties from observations using a Roman- or Habitable Exoplanet Observatory (HabEx)-like telescope, comparing the strategies to observe at multiple orbital phases or in multiple wavelength bands. With a $\sim 20 \%$ bandwidth in $600-800 \mathrm{~nm}$ on a Roman-like telescope, the retrieval finds a degenerate scenario with a lower gas abundance and a deeper or absent cloud than the truth. Repeating the observation at a different orbital phase or at a second $20 \%$ wavelength band in $800-1000 \mathrm{~nm}$, with the same integration time and thus degraded signal-to-noise ratio $(\mathrm{S} / \mathrm{N})$, would effectively eliminate this degenerate solution. Single observation with a HabEx-like telescope would yield high-precision constraints on the gas abundances and cloud properties, without the degenerate scenario. These results are also generally applicable to high-contrast spectroscopy with a coronagraph with a similar wavelength coverage and $\mathrm{S} / \mathrm{N}$, and can help design the wavelength bandwidth and the observation plan of exoplanet direct-imaging experiments in the future.
\end{abstract}

Unified Astronomy Thesaurus concepts: Astronomical techniques (1684); Direct imaging (387); Spectroscopy (1558); Exoplanet atmospheres (487); Exoplanet atmospheric composition (2021); Bayesian statistics (1900); Posterior distribution (1926)

\section{Introduction}

Direct imaging of exoplanets has begun to pick up momentum as a way to characterize exoplanetary atmospheres. High-contrast observations from the ground have measured thermal emission spectra of several exoplanets (Janson et al. 2010; Konopacky et al. 2013; Macintosh et al. 2015; Wagner et al. 2016; Rajan et al. 2017; Samland et al. 2017), and the spectra have shown the presence of carbon monoxide and water in their atmospheres. These planets are young giant planets so that they emit detectable thermal emission despite being well separated from their host stars.

Spaceborne direct-imaging capabilities in the future will enable atmospheric characterization in the reflected light. The Nancy Grace Roman Space Telescope (Roman hereafter, previously know as Wide-Field InfraRed Survey Telescope; Spergel et al. 2015; Akeson et al. 2019) will be capable of collecting starlight reflected by giant exoplanets through highcontrast imaging. The Starshade Rendezvous with Roman (Seager et al. 2019), an advanced mission concept, would further enable Roman to reach a planet-star contrast ratio as low as $\sim 10^{-10}$. The Habitable Exoplanet Observatory (HabEx; Gaudi et al. 2020), a concept of a $4 \mathrm{~m}$ space telescope with a starshade, has the main objective to image potentially Earthlike planets and study their atmospheres. Starshade Rendezvous and HabEx, both with a starshade, would allow for the study of giant exoplanets orbiting their host stars at about 1-10 au with reflected light spectroscopy.
Reflected light spectra from these cold gaseous planets contain rich information on the chemical composition and cloud formation in their atmospheres (e.g., Sudarsky et al. 2000, 2003; Burrows et al. 2004; Cahoy et al. 2010; Lupu et al. 2016; MacDonald et al. 2018; Hu 2019; Damiano \& Hu 2020; Carrión-González et al. 2020). When the wavelength coverage is limited and with moderate wavelength resolution and signalto-noise ratio $(\mathrm{S} / \mathrm{N})$, there can be substantial degeneracy between the atmospheric abundance and the cloud pressure (e.g., Lupu et al. 2016; Nayak et al. 2017; Hu 2019; CarriónGonzález et al. 2020). In short, a deep cloud and a small abundance of an absorber (e.g., $\mathrm{CH}_{4}$ ) may result in a similar spectrum as a shallower cloud and a higher abundance of absorber, leading to the degeneracy.

A potential strategy to break the degeneracy is to observe the planets at multiple orbital phase angles. This is because viewing the same atmosphere at different phase angles would produce a wavelength-dependent difference in the spectra as the illumination and emerging angles have chromatic effects in atmospheric absorption and cloud reflection (Cahoy et al. 2010; Madhusudhan \& Burrows 2012; Nayak et al. 2017).

The reflected light spectra of Jupiter measured by telescopes from the center of the planet to the limb has led to constraints of the position and the vertical extent of the planet's upper cloud layer (e.g., Sato \& Hansen 1979). Whether a similar measurement is as informative for exoplanets-disk integrated but viewed at different phase angles-is not well understood. A previous study (Nayak et al. 2017) was focused on modeling the relationship between the phase angle and other parameters 
such as cloud properties, methane abundance, and planetary radius by considering the Roman coronagraph setup. Taking into account single observations, the authors found that the knowledge of a precise phase angle does not improve the estimates for methane and cloud properties. It instead helps to improve the constraints on the planetary radius by a factor of 2 . In that study, the authors considered the combination of observations at different phase angles using the intersection criterion-a practical approximation of the exploration of parameter space without employing a Bayesian statistical search. Finally, they concluded that multiple observations at different phase angles might be beneficial for a better constraint on the physical/chemical planetary parameters.

Here, we evaluate the multi-orbital-phase observations as a strategy to refine the constraints on the atmospheric properties from reflected light spectroscopy, and compare it to multiwavelength-band observations with a starshade. We focused our attention on the realistic noise model of the instruments ( i.e., telescope plus starshade) and on possible degenerate scientific interpretations of the results. Recognizing realistic operational overheads (e.g., the starshade would need to reposition itself precisely between the telescope and the star and this maneuver costs fuel), we specifically determine which of the following would produce the optimal science return when repeated observations are possible for an object of interest: (1) repeat the observation in the same band but at a different phase, (2) repeat the observation at the same phase but in a second band, and (3) repeat the observation at the same phase and in the same band. We use the Starshade Imaging Simulation Toolkit for Exoplanet Reconnaissance ${ }^{4}$ (SISTER) to simulate data and uncertainties obtained in starshade-enabled spectroscopic observations. We then use a robust Bayesian inverse retrieval method (EXOREL ${ }^{\Re}$; Damiano \& Hu 2020) to determine and compare the information rendered by the three strategies.

The paper is organized as follows. Section 2 describes the simulation of the observed spectra and the multiphase spectral retrieval method, Section 3 shows the results and compares the posterior constraints among the observation scenarios, and Section 4 discusses the observational strategy and summarizes the key findings of this study.

\section{Methods}

\subsection{Simulated Planet Spectra}

We use 47 UMa b, a giant planet orbiting a G0V star at $2.1 \mathrm{au}$, as the representative planet in this study. $47 \mathrm{UMa} \mathrm{b}$ is in the design reference mission of both Starshade Rendezvous (Seager et al. 2019) and HabEx (Gaudi et al. 2020). Compared to Jupiter, 47 UMa b orbits closer to its host star and has a higher equilibrium temperature. In terms of cloud structure, this means that the upper atmosphere of the planet is expected to contain water clouds, as opposed to the ammonia clouds typical of Jupiter (Sudarsky et al. 2000). We simulated the atmospheric abundance and cloud structure of this exoplanet in our previous work (Damiano \& Hu 2020). Here we use EXOREL (Hu 2019; Damiano \& Hu 2020) to synthesize the reflected light spectra at the orbital phases of $\pi / 3$ and $\pi / 2$. These phases are picked because the phase of $\pi / 3$ is a good compromise between the angular separation between the planet and the star and the

\footnotetext{
http://sister.caltech.edu
}

brightness in the reflected light (and thus the go-to phase of a single observation). The phase of $\pi / 2$ is when the angular separation between the star and the planet is maximized. Should the planet be a Lambertian sphere, the brightness at $\pi / 2$ is $\sim 50 \%$ of that at $\pi / 3$; but our model calculates the wavelength-dependent phase function produced by clouds and gases in the atmosphere. It is assumed in this work that the planet's atmosphere is the same at all longitudes, i.e., the clouds are not localized and the physical structure of the cloud does not change between phases.

\subsection{Simulated Uncertainties}

A novel aspect of this study is the inclusion of realistic uncertainties expected from observations using a starshade. We use SISTER (S. R. Hildebrandt et al. 2020, in preparation) to simulate the photons' trajectory in the starshade-telescope system as well as detector effects in the telescope. Exposure time calculators (e.g., Robinson et al. 2016) have been developed to estimate the uncertainties of a starshade or coronagraph observation. However, they did not take into account the full two-dimensional nature of the astrophysical scene or the spatial variation of the point-spread function due to the optical diffraction from the starshade. SISTER contemplates these aspects. Most details of SISTER can be found in its handbook. ${ }^{5}$ Our simulated astrophysical scene includes 47 UMa b, residual starlight, exozodiacal light (five times dustier than the solar system; Ertel et al. 2020), solar glint (i.e., scattered and reflected light from the Sun by the starshade; Hilgemann et al. 2019), and local zodiacal light. Specifically, we consider petals of the starshade to have imperfection compatible with current lab work, which results in a greater residual starlight than an ideal starshade. We then place the planet as close as possible to the maximum intensity of the solar glint compatible with its orbital parameters. As such, we generate a scenario that serves as an upper limit for the integration time compared to other more favorable scenarios of the same system. Figure 1 shows the (noiseless) contribution of each component arriving at the detector.

On the telescope side, the optical throughput and the quantum efficiency $(\mathrm{QE})$ of the detector follow the expected performance of Roman and HabEx, respectively. The detector models an electron multiplying charge couple device (EMCCD). The EMCCD gain factor is set to 1000, making the read-out noise effectively zero. We choose noise parameters corresponding to those adopted by Roman and HabEx, respectively, including clock-induced charge and dark current (Nemati et al. 2017).

When computing the uncertainties in the planetary counts, we assume that residual starlight, solar glint, exozodiacal light, and local zodiacal light can be subtracted out with some postprocessing technique to the shot-noise limit, i.e., leaving no systematic bias in the measurements. This assumption increases the shot noise of these components by $\sim \sqrt{2}$ as a consequence of subtracting a noisy template from noisy data. The final error bars for each spectral bin are then derived from 1000 independent SISTER simulations and used to generate independent Gaussian noise realizations, which are finally added to the input spectral data points.

We now turn to the specific scenarios that correspond to Roman and HabEx.

\footnotetext{
5 http://sister.caltech.edu
} 

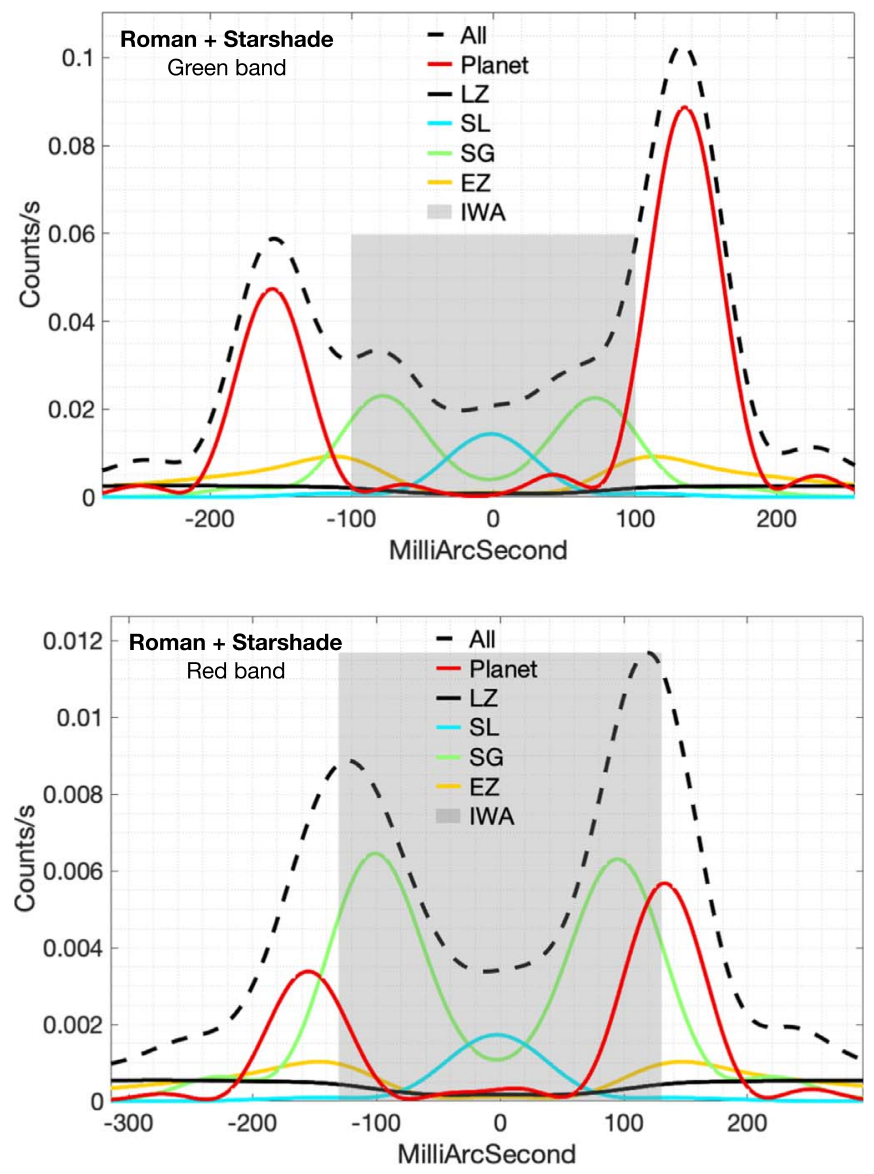

Figure 1. Contribution of each astrophysical and background component to the Starshade Rendezvous simulation performed by SISTER. The unit on the vertical axis is the rate of photons arriving at the instrument's detector taking into account all the optical and instrumental effects, except noise, and has been integrated over the bandwidth. All is the sum of all individual contributions. Planet refers to the rate coming from the planet only. We plot on the left-hand side the planet when its orbital phase is $\pi / 2$, and on the right-hand side when it is $\pi / 3$. LZ refers to the contribution from the local zodiacal light at $22.5 \mathrm{~V}$ $\mathrm{mag} / \operatorname{arcsec}^{2}$. SL is the contribution of the residual starlight from starshade imperfections. SG refers to the contribution from the solar glint for a representative angle of the Sun with respect to the target star. EZ is the contribution from the exozodiacal emission, which was chosen to be five times the dust density of the solar system. Finally, IWA refers to the geometric inner working angle and is the apparent size of the starshade as seen by the telescope. Diffraction effects are relevant close and inside the IWA. Top: $0.61-0.75 \mu \mathrm{m}$. The planet-star flux ratio averaged over the bandwidth is $1.9 \times 10^{-8}$ when the orbital phase is $\pi / 3$ and $1.0 \times 10^{-8}$ when it is $\pi / 2$. The IWA is 100.2 mas. Bottom: $0.82-1.0 \mu \mathrm{m}$. The average planet-star flux ratio is $7.6 \times 10^{-9}$ when the orbital phase is $\pi / 3$ and $4.2 \times 10^{-9}$ when it is $\pi / 2$. The IWA is 130.4 mas. Notice that the photon rate is lower than in the $0.61-0.75 \mu \mathrm{m}$ band.

\subsubsection{Starshade Rendezvous with Roman}

We consider a Roman-like telescope with an integral field spectrograph (IFS) that operates in three separate wavelength bands in $0.4-1.0 \mu \mathrm{m}$ with a $20 \%$ bandwidth. We specifically assume blue $(0.45-0.55 \mu \mathrm{m})$, green $(0.61-0.75 \mu \mathrm{m})$, and red $(0.82-1.0 \mu \mathrm{m})$ bands, all with a resolving power of $R=50$. These bands do not match exactly with the Roman coronagraph's baseline design (V. Bailey 2020, private communication) or the design reference mission of the Starshade Rendezvous concept (Seager et al. 2019), as these designs have been evolving. In general, however, the blue band would cover the expected brightest section of the albedo spectrum and so is the best for planet detection, the green band would cover a strong absorption band of $\mathrm{CH}_{4}$ and so is the primary band for atmospheric characterization, and the red band would provide additional information on $\mathrm{CH}_{4}$ and cover an absorption band of $\mathrm{H}_{2} \mathrm{O}$ (e.g., Lupu et al. 2016; MacDonald et al. 2018). With a $20 \%$ bandwidth, multiple observations would be necessary to obtain a spectrum that spans more than one band.

We determine the integration time by requiring an average $\mathrm{S} / \mathrm{N}$ of 20 per spectral bin of the blue band when the orbital phase is $\pi / 3$. The integration time is $\sim 3.5 \mathrm{hr}$. We use this integration time in the other two bands, and in the observations with an orbital phase of $\pi / 2$. We obtain an average $\mathrm{S} / \mathrm{N}$ of 18.0 per spectral bin in the green band and 2.3 in the red band when the orbital phase is $\pi / 3$. The $\mathrm{S} / \mathrm{N}$ decreases to 11.5 and 1.5 when the orbital phase is $\pi / 2$ (see Figure 3 ). The ratio of $\mathrm{S} /$ $\mathrm{N}$ between the $60^{\circ}$ observation and the $90^{\circ}$ observation is $\sim 1.55$ while the ratio of planetary flux is $\sim 2$. This is because the planetary flux contributes substantially to the shot noise in these observations, and the background fluxes from solar glint and exozodiacal dust decrease for wider angular separations from $\pi / 3$ to $\pi / 2$ (Figure 1 ).

The integration times estimated here do not include the expected difference in detector $\mathrm{QE}$, dark current, and clockinduced charge between beginning of life (BOL) and end of life (EOL). Roman is expected to be launched before the Starshade Rendezvous. Therefore, the QE, the dark current, and the clock-induced charge will degrade by the time the Starshade is coupled with Roman. If we take into account these factors, the calculated integration time would be $\sim 8.7 \mathrm{hr}$, compared with $\sim 3.5 \mathrm{hr}$. These achromatic factors do not introduce substantial differences in the $\mathrm{S} / \mathrm{N}$ ratio between different wavelength bands and phase angle observations.

\subsection{2. $H a b E x$}

The HabEx concept (Gaudi et al. 2020) entails a larger telescope than Roman that would be able to obtain spectra in a broad wavelength range between 0.45 and $1.0 \mu \mathrm{m}$ with a resolving power of $R=140$ with a single observation. The broader bandwidth and higher spectral resolution may provide improved constraints on atmospheric properties. The HabEx concept also entails a larger starshade than the Roman Rendezvous, and it would be placed further away from the telescope. This results in a decrease of the solar glint background, and this effect is included in SISTER. Here we evaluate whether multiphase observations would further improve the constraints from single observation. The HabEx design includes an additional wavelength band at $1.0-1.8 \mu \mathrm{m}$; we do not include this band in the analysis because it is primarily for characterizing small planets (Gaudi et al. 2020).

We simulate the uncertainties in HabEx simulations in a similar way as with Roman. To compare the two cases, we adjust the integration time to obtain an average $S / N$ of 20 per spectral bin in the $0.45-0.55 \mu \mathrm{m}$ interval when the orbital phase is $\pi / 3$. The integration time is $\sim 1.8 \mathrm{hr}$. As in the case of Roman, we use this integration time when the orbital phase is $\pi / 2$. The average $\mathrm{S} / \mathrm{N}$ per spectral bin in the $0.45-0.55 \mu \mathrm{m}$ interval is 15.0 when the orbital phase is $\pi / 2$ (see Figure 4).

We note that despite the fact that the HabEx design is expected to have higher spectral resolution, larger primary mirror diameter, and better QE above $750 \mathrm{~nm}$, the integration time estimated here is only a factor of $\sim 2$ less than the Roman scenario because we based our calculations at $500 \mathrm{~nm}$ where the QE of the two instruments is similar. We then used the 
Table 1

Results of the Retrievals for Each of the Observational Scenarios of Starshade Rendezvous with Roman Considered for 47 UMa b

\begin{tabular}{|c|c|c|c|c|c|}
\hline \multirow{2}{*}{ Parameters } & \multirow{2}{*}{ Truths } & \multicolumn{4}{|c|}{ Starshade Rendezvous with Roman } \\
\hline & & $\begin{array}{c}\text { Green Band } \\
\alpha=\pi / 3 \text { only }\end{array}$ & $\begin{array}{c}\text { Green Band } \\
\alpha=\pi / 3 \& \pi / 2\end{array}$ & $\begin{array}{c}\text { Green and Red Bands } \\
\qquad \alpha=\pi / 3\end{array}$ & $\begin{array}{c}\text { Green Band } \\
\alpha=\pi / 3 \text { twice }\end{array}$ \\
\hline $\log \left(\mathrm{NH}_{3}\right)$ & -2.36 & $-7.46_{-4.16}^{+6.18}$ & $-6.29_{-5.15}^{+5.65}$ & $-4.50_{-6.64}^{+3.55}$ & $-6.90_{-4.72}^{+5.49}$ \\
\hline $\log \left(\mathrm{CH}_{4}\right)$ & -1.80 & $-1.45_{-2.61}^{+1.13}$ & $-1.36_{-1.61}^{+0.93}$ & $-1.43_{-1.67}^{+1.02}$ & $-1.52_{-1.12}^{+1.17}$ \\
\hline $\log \left(P_{\mathrm{H}_{2} \mathrm{O}}[\mathrm{Pa}]\right)$ & 3.84 & $2.70_{-2.43}^{+4.62}$ & $3.05_{-2.13}^{+1.78}$ & $3.29_{-2.15}^{+1.93}$ & $3.53_{-2.85}^{+1.42}$ \\
\hline $\log \left(g\left[\mathrm{~cm} \mathrm{~s}^{-2}\right]\right)$ & 3.65 & $3.66_{-0.04}^{+0.05}$ & $3.68_{-0.03}^{+0.03}$ & $3.68_{-0.04}^{+0.03}$ & $3.66_{-0.06}^{+0.03}$ \\
\hline
\end{tabular}

Note. $\alpha$ is the phase angle. The reported error bars are relative to the $95 \%$ confidence interval.

same integration time for other wavelengths to focus our attention on the $\mathrm{S} / \mathrm{N}$ ratio between different wavelengths. The benefits of HabEx are appreciated at longer wavelengths where the differences between the two instruments are substantial (see Section 3 and Figure 4).

\subsection{Multiphase and Multiband Retrieval}

We augment the EXOREL ${ }^{\Re}$ retrieval framework (Damiano \& Hu 2020) with the ability to retrieve multiple spectra taken at different phase angles or in different wavelength bands. To simultaneously fit the spectra within the same Bayesian instance, we calculate the likelihood of each set of data and take the product. Specifically, when a set of free parameters is chosen to be evaluated, we calculate the spectrum for the first phase angle and we compare the model with the data to obtain the likelihood $\mathcal{L}_{1}$. With the same set of parameters we calculate the model referred to as the second phase angle and we compare it to the data to calculate the likelihood $\mathcal{L}_{2}$. The product, $\mathcal{L}_{1} \times \mathcal{L}_{2}$, will give the total likelihood of the free parameters set as

$$
\log (\mathcal{L})=\log \left(\mathcal{L}_{1} \times \mathcal{L}_{2}\right)=\log \left(\mathcal{L}_{1}\right)+\log \left(\mathcal{L}_{2}\right)
$$

Another update is that we now retrieve from the planet-star contrast ratio rather than the albedo in Damiano \& Hu (2020), because the uncertainties simulated are expressed in the planetstar contrast ratio. This brings in the planetary radius as a major factor. We now include the surface gravity as an additional free parameter in the retrieval, and the planetary radius is derived from the planetary mass and the retrieved surface gravity.

\section{Results}

The constraints on the atmospheric properties retrieved from the simulated observations with Starshade Rendezvous with Roman and HabEx are summarized in Tables 1 and 2 and shown in Figures 3 and 4. Definitions of these parameters and their impact on the albedo spectrum are described in detail in Damiano \& $\mathrm{Hu}$ (2020). Briefly, $\log \left(\mathrm{H}_{2} \mathrm{O}\right), \log \left(\mathrm{NH}_{3}\right)$, and $\log \left(\mathrm{CH}_{4}\right)$ are the mixing ratios of the trace gases; since we include condensation of $\mathrm{H}_{2} \mathrm{O}$, the mixing ratio of $\mathrm{H}_{2} \mathrm{O}$ is the mixing ratio below the cloud. $\log \left(P_{\mathrm{H}_{2} \mathrm{O}}\right)$ and $\log \left(D_{\mathrm{H}_{2} \mathrm{O}}\right)$ are the top pressure and depth of the $\mathrm{H}_{2} \mathrm{O}$ cloud. The cloud depth is the difference in pressure between the bottom and the top of the cloud. $\log \left(\mathrm{CR}_{\mathrm{H}_{2} \mathrm{O}}\right)$ is the ratio between the mixing ratio of $\mathrm{H}_{2} \mathrm{O}$ above the cloud and that below the cloud-the difference is condensed out to form the cloud.
Table 2

Results of the Retrievals for Each of the Observational Scenarios of HabEx Considered for $47 \mathrm{UMa} b$

\begin{tabular}{|c|c|c|c|c|}
\hline \multirow{2}{*}{ Parameters } & \multirow{2}{*}{ Truths } & \multicolumn{3}{|c|}{ HabEx } \\
\hline & & $\begin{array}{c}\alpha=\pi / 3 \\
\text { only }\end{array}$ & $\begin{array}{c}\alpha=\pi / 3 \text { and } \\
\alpha=\pi / 2\end{array}$ & $\begin{array}{c}\alpha=\pi / 3 \\
\text { twice }\end{array}$ \\
\hline $\log \left(\mathrm{H}_{2} \mathrm{O}\right)$ & -1.50 & $-1.60_{-0.31}^{+0.58}$ & $-1.32_{-0.40}^{+0.78}$ & $-1.30_{-0.37}^{+0.71}$ \\
\hline $\log \left(\mathrm{NH}_{3}\right)$ & -2.36 & $-2.78_{-8.51}^{+0.98}$ & $-2.11_{-6.43}^{+0.91}$ & $-2.51_{-8.36}^{+0.82}$ \\
\hline $\log \left(\mathrm{CH}_{4}\right)$ & -1.80 & $-1.76_{-0.36}^{+0.36}$ & $-1.59_{-0.33}^{+0.61}$ & $-1.62_{-0.19}^{+0.51}$ \\
\hline $\log \left(P_{\mathrm{H}_{2} \mathrm{O}}[\mathrm{Pa}]\right)$ & 3.84 & $2.52_{-1.95}^{+1.50}$ & $2.22_{-1.95}^{+1.48}$ & $2.44_{-1.74}^{+1.34}$ \\
\hline $\log \left(D_{\mathrm{H}_{2} \mathrm{O}}[\mathrm{Pa}]\right)$ & 4.97 & $5.00_{-0.16}^{+0.24}$ & $4.96_{-0.24}^{+0.16}$ & $4.96_{-0.16}^{+0.07}$ \\
\hline $\log \left(\mathrm{CR}_{\mathrm{H}_{2} \mathrm{O}}\right)$ & -5.04 & $-8.75_{-3.04}^{+4.94}$ & $-9.21_{-2.65}^{+4.77}$ & $-9.18_{-2.69}^{+4.88}$ \\
\hline $\log \left(g\left[\mathrm{~cm} \mathrm{~s}^{-2}\right]\right)$ & 3.65 & $3.65_{-0.01}^{+0.02}$ & $3.66_{-0.01}^{+0.02}$ & $3.66_{-0.01}^{+0.02}$ \\
\hline
\end{tabular}

Note. $\alpha$ is the phase angle. The reported error bars are relative to the $95 \%$ confidence interval.

Roman. The most plausible starting point to characterize the atmosphere of 47 UMa $b$ in reflected light is a single observation in the green band. Our retrieval analysis in this scenario finds a solution close to the truth, as well as a degenerate solution (dashed blue lines in Figures 2 and 3). While the truth has a mixing ratio of $\mathrm{CH}_{4}$ of $\sim 10^{-2}$ and $\mathrm{a}_{2} \mathrm{O}$ cloud at $0.1 \sim 1$ bar, the degenerate solution has a mixing ratio of $\mathrm{CH}_{4}$ of $\sim 10^{-4}$ and corresponding mixing ratio of $\mathrm{H}_{2} \mathrm{O}<$ $10^{-6}$ (Figure 3). With such a low abundance of water, there is not enough mass to form a reflective cloud, and so the degenerate solution is insensitive to the cloud description parameters $\left(\log \left(P_{\mathrm{H}_{2} \mathrm{O}}\right), \log \left(D_{\mathrm{H}_{2} \mathrm{O}}\right)\right.$, and $\left.\log \left(\mathrm{CR}_{\mathrm{H}_{2} \mathrm{O}}\right)\right)$. The degenerate solution is essentially a cloud-free atmosphere with the mixing ratio of $\mathrm{CH}_{4}$ two orders of magnitude lower than the truth.

Adding a second observation at a different orbital phase ( $\alpha=\pi / 2$ in this study) with the same integration time would drastically reduce the likelihood of the degenerate solution. The $\mathrm{S} / \mathrm{N}$ of the second observation is poorer, because the baseline of the spectrum is lower at the phase of $\alpha=\pi / 2$ than at the phase of $\alpha=\pi / 3$. The simultaneous retrieval of the observations at both phases would find the true result (see the orange model and distributions shown in Figure 3). The improvement in the constraints comes from two factors. First, adding a second observation inevitably increases the $\mathrm{S} / \mathrm{N}$ of the overall observation, which helps eliminate the degenerate solution. Second, the true and the degenerate scenarios have different achromatic phase functions, and the biggest difference occurs 

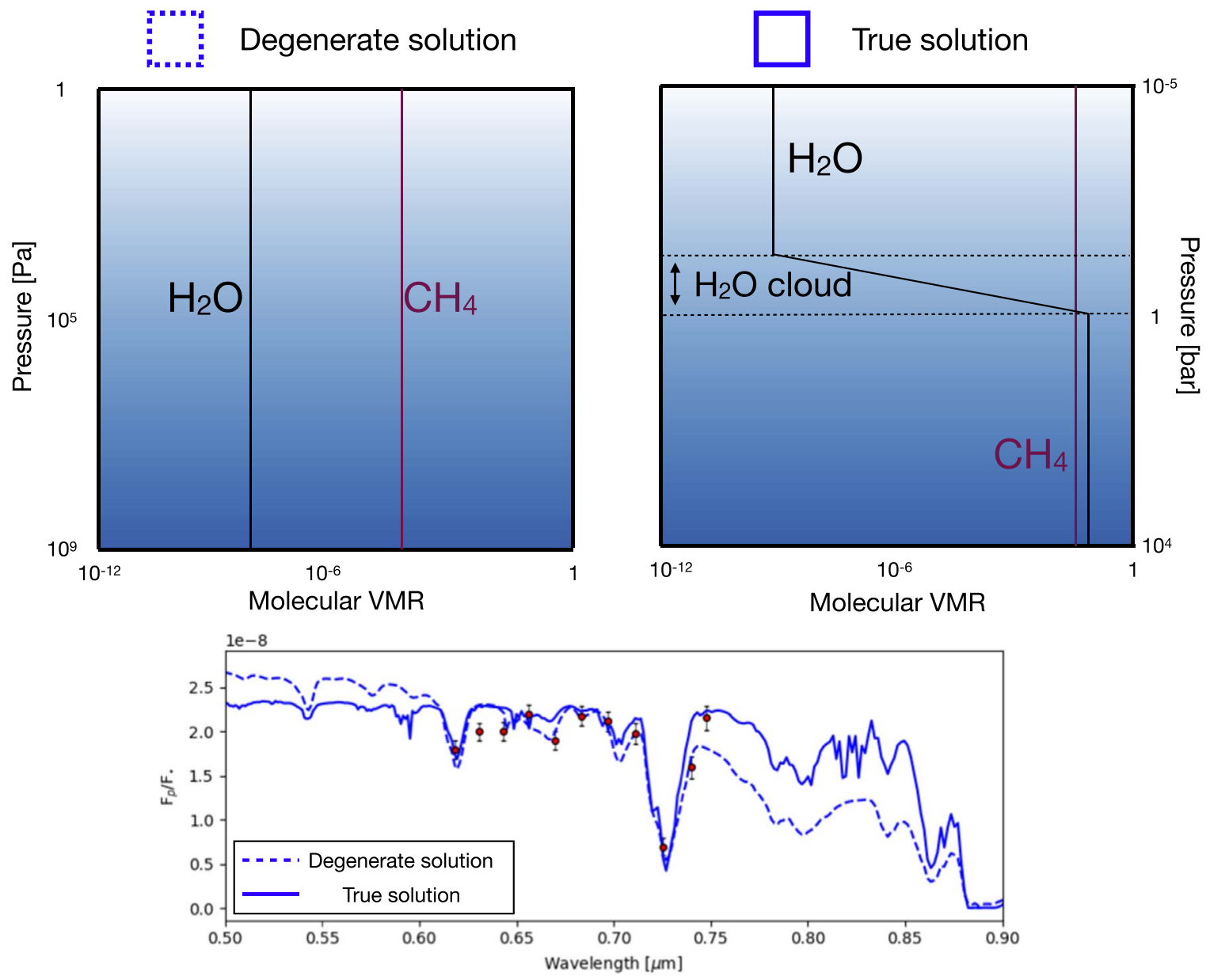

Figure 2. Top panels: atmospheric composition of the degenerate solution (on the left) and the true solution (on the right). The degenerate solution represents a clear sky scenario with a low abundance of water and methane. The true solution, on the other hand, comprises a water cloud at around 1 bar. Water and methane concentrations are higher than the degenerate solution. Bottom panel: the spectra of the two scenarios (dashed line for the degenerate scenario and solid line for the true one) are represented together with the simulated data (green band, $\alpha=\pi / 3$ ). The difference between the two spectra is large at shorter and longer wavelengths.

at the main absorption bands (Figure 3). The difference is on the order of $5 \%$, so that the $\mathrm{S} / \mathrm{N}$ of 11.5 per spectral bin from the assumed observational scenario could partly leverage this diagnostic power.

Moreover, adding an observation in the red band with the same integration time would also drastically reduce the significance of the degenerate solution almost to zero. Here we assume that the second observation takes place immediately after the first one, and they essentially correspond to the same orbital phase. The red -band observation has an average $\mathrm{S} / \mathrm{N}$ of only $\sim 2$, due to decreasing starlight intensity, lower planetary albedo, stronger solar glint, and degrading detector QE from the green band to the red band. Despite the poor $\mathrm{S} / \mathrm{N}$ of the red-band spectrum, we find it highly informative when combined with the green-band spectrum. The retrieval of the observations at both bands does not show a degenerate solution (see the green model and distributions shown in Figure 3). This is because the degenerate scenario has a much lower albedo than the truth in the red band, and the difference is well on the order of $30 \%$ in $0.75-0.85 \mu \mathrm{m}$.

The last scenario considered is to repeat the first observation at the same wavelength band and at the same orbital phase, i.e., a second realization. The combined $\mathrm{S} / \mathrm{N}$ is $\sim 25$ per spectral bin, and to our surprise, this strategy would also eliminate the degenerate solution. Most of the diagnosing power comes from the datum at $0.75 \mu \mathrm{m}$, where the truth and the degenerate scenario differ the most.

As long as the degenerate solution is eliminated, the reflected light spectral retrieval can constrain not only the mixing ratio of $\mathrm{CH}_{4}$ in the atmosphere, but also the mixing ratio of $\mathrm{H}_{2} \mathrm{O}$ below the cloud (as it is the feedstock for cloud), and the pressure level of the cloud. There is a moderate correlation between the mixing ratio of $\mathrm{CH}_{4}$ and the pressure level of the cloud (Figure 3). Inferring the mixing ratio of $\mathrm{H}_{2} \mathrm{O}$ below the cloud is possible because our retrieval method preserves the causal relationship between the condensation of water vapor and the formation of a water cloud (Damiano \& Hu 2020). The surface gravity, and thus the radius of the planet, is constrained in all scenarios.

HabEx. A single observation of HabEx with an $\mathrm{S} / \mathrm{N}$ of $\sim 20$ would pinpoint the atmospheric properties (see the blue model and distributions shown in Figure 4). Specifically, the mixing ratios of $\mathrm{CH}_{4}$ and $\mathrm{H}_{2} \mathrm{O}$ would be well constrained to the precision of $\sim 1 / 3 \mathrm{dec}$, and that of the cloud pressure would be better constrained than $\sim 1 / 5 \mathrm{dec}$. There is no correlation between the mixing ratio of $\mathrm{CH}_{4}$ and the cloud pressure in this case. Notably, the spectrum yields meaningful constraints on the mixing ratio of $\mathrm{NH}_{3}$, mostly from the small absorption feature at $\sim 0.63 \mu \mathrm{m}$. These improvements come from not only a broader wavelength coverage and a higher spectral resolution 


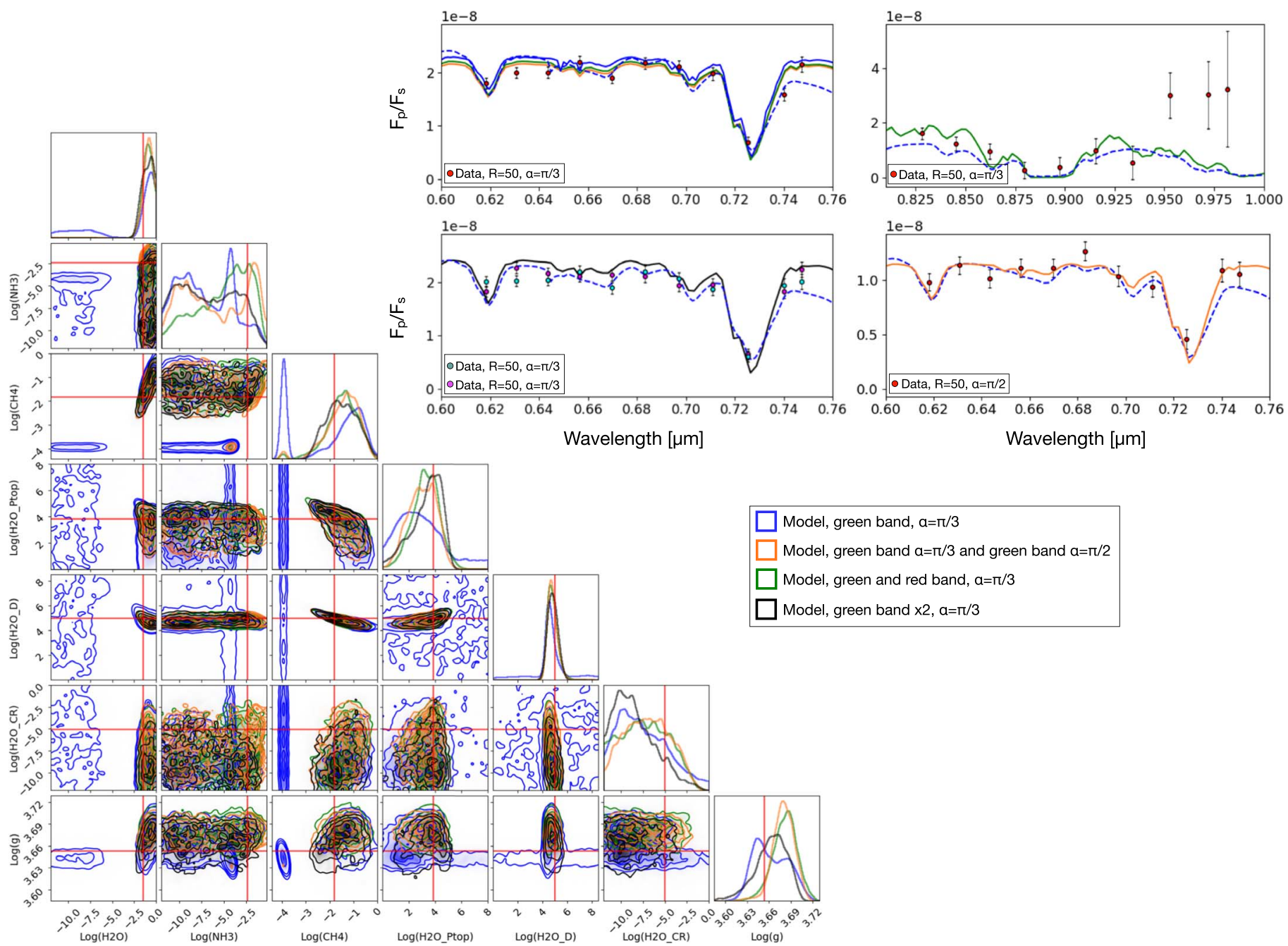

Figure 3. Retrieval analysis of the four observational scenarios of Starshade Rendezvous with Roman. The four panels on the top show the best-fit models and the simulated data. The dashed blue line in the first panel shows the degenerate solution obtained with a single observation in the green band and at the orbital phase of $\pi /$ 3; the other three panels show how this solution may be inconsistent with additional observations. The corner plot on the bottom shows the marginalized posterior distribution for each of the parameters, using the same color scheme to denote the four observational scenarios simulated. The degenerate solution is eliminated with a second observation at the same phase, at a wider-separated phase of $\pi / 2$, or in the red band.

than Roman, but also the larger and further starshade (for lower solar glint) and the larger telescope aperture (for lower exozodiacal light). Adding another observation with at the same or a different orbital phase would slightly reduce uncertainty in the retrieved mixing ratios of $\mathrm{CH}_{4}$, while the overall gain in the constraints of the parameters is minimal.

\section{Discussion and Conclusion}

With the case studies presented in Section 3, we show that with a limited wavelength band like the green band on Roman, a single observation of a planet's reflected light spectrum at the favorable orbital phase (e.g., $\alpha=\pi / 3$ ) and a high $\mathrm{S} / \mathrm{N}$ (e.g., $\sim 20)$ can result in degenerate solutions. The degeneracy is between the atmospheric scenario of a higher cloud and greater mixing ratio of $\mathrm{CH}_{4}$ versus the scenario of a lower or absent cloud and smaller mixing ratio of $\mathrm{CH}_{4}$. This type of degeneracy has been discussed in $\mathrm{Hu}(2014,2019)$ and it is shown here with realistic noise estimates and rigorous retrieval.

Expanding the wavelength coverage to longer wavelengths ( i.e., the red band), doubling the integration time, or repeating the observation at a different orbital phase with a similar integration time would be able to eliminate this degeneracy. As shown in Section 3, these three strategies result in similar quality constraints on the atmospheric parameters.

Particularly, the spectra that are degenerate in the green band diverge at longer wavelengths: the low or absent cloud scenario would have a much lower albedo than the high cloud scenario. The divergence is often so significant that even a low $\mathrm{S} / \mathrm{N}$ observation at the long wavelengths can effectively eliminate the degenerate solution. To date, it is often assumed that the red-band observation with Roman would be severely affected by the degrading QE of the detector in this band (e.g., Seager et al. 2019). Here we show that even with the poor QE and other complications, a red-band observation with the same integration time as the green band can be highly informative. This finding also implies that the constraints on the atmospheric properties would be drastically improved at no additional integration time, if the wavelength bandwidth could be enlarged to cover both the green band and the red band with a single observation.

If broadening the wavelength coverage is not possible, eliminating the degenerate solution would require an $\mathrm{S} / \mathrm{N}$ per spectral bin of at least $\sim 25$, which may be achieved by 


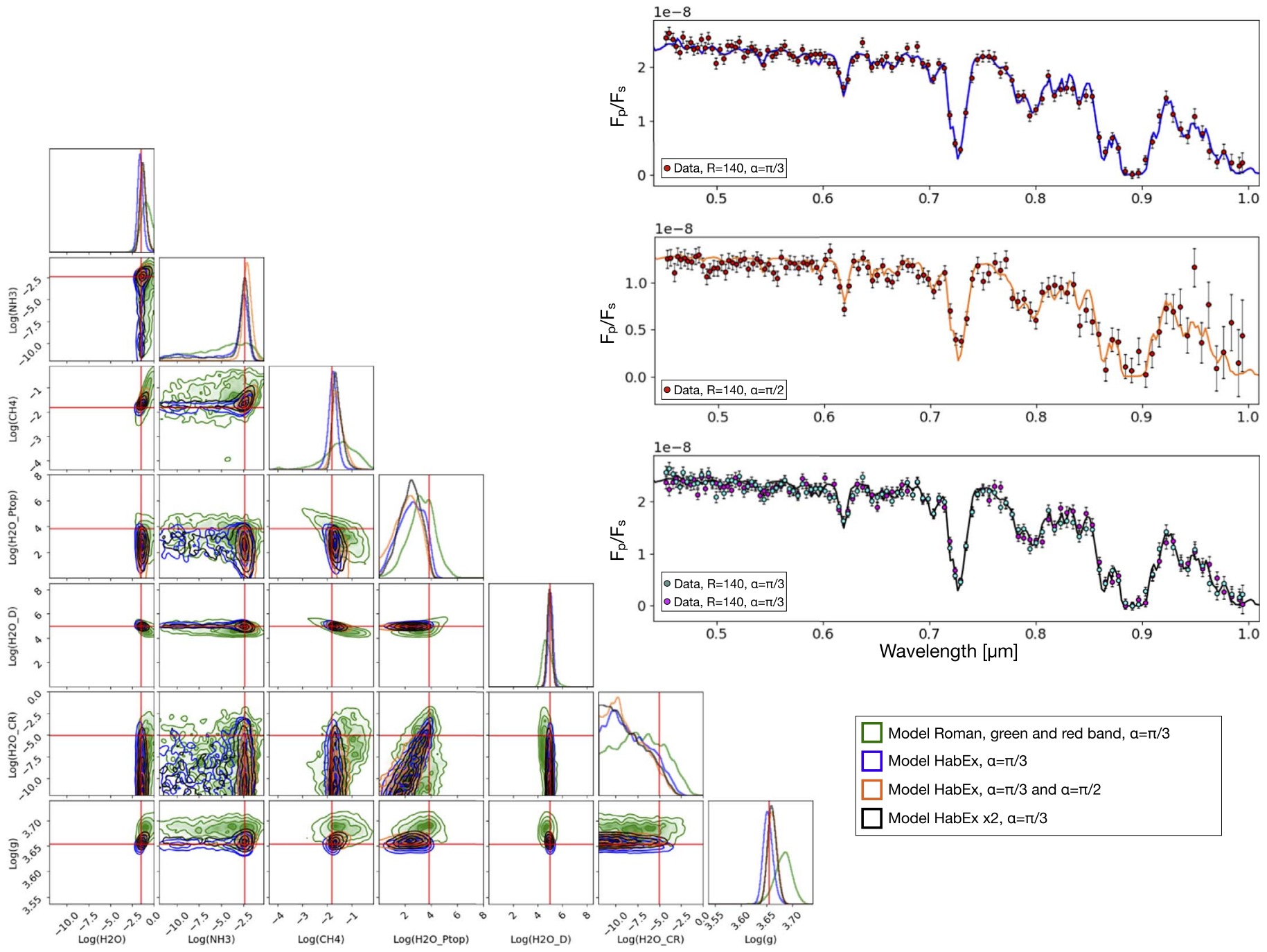

Figure 4. Retrieval analysis of the three observational scenarios of HabEx. The three panels on the top show the best-fit models and the simulated data. The corner plot on the bottom shows the marginalized posterior distribution for each of the parameters, using the same color scheme to denote the three observational scenarios simulated. The green model in the corner plot is the same as the green model in Figure 3, and it is shown for comparison. A single observation with HabEx would be sufficient to constrain the atmospheric properties.

integrating longer in a single observation or revisiting the same planet at a different orbital phase. At an $\mathrm{S} / \mathrm{N}$ of $\sim 18$ per visit, the difference between revisiting and integrating longer is minimal, and thus integrating longer may be the preferred strategy to avoid re-targeting the starshade. As shown in Figure 3, there is indeed a difference in the spectral features between the two phases, consistent with Cahoy et al. (2010). To capture this subtle difference, however, will require a high $\mathrm{S} / \mathrm{N}(>20)$ on the observations at both orbital phases and thus a longer integration time for the observation at the less-thanoptimal phase. The analysis here highlights that, when only one observation in a limited wavelength bandwidth is expected, it is important to schedule that observation at the phase that maximizes the $\mathrm{S} / \mathrm{N}$ of the spectrum and with sufficient duration.

In this work, we have assumed the phase angle of the observations and the mass of the planet to be known. In this context, the planetary gravity, which we considered as a retrievable parameter, is effectively derived from the radius of the planet. This assumption for the characterization of giant planets is acceptable as observational campaigns and radial velocity observations may provide a good constraint on the aforementioned parameters. However, if the phase angle and/ or the mass of the planet are poorly known, the planetary radius would become a free parameter detached from the gravity, and one would expect correlations between the planetary radius and the phase angle (Nayak et al. 2017).

In this project, when considering multiphase-observations and multiband-observations, we focused our attention on the noise ratio between the scenarios using the same integration time for all the observations. This means that in this work we combined observations very different in terms of $\mathrm{S} / \mathrm{N}$. In spite of this fact, we obtained improvements on the constraint of the free parameters when multiple observations have been combined. In line with previous work (Nayak et al. 2017), the improvements generally do not exceed the order of magnitude, with the most significant improvements in the methane abundance and in the cloud top pressure constraints.

With a wide wavelength coverage in the visible and nearinfrared, a HabEx-like telescope would provide precise constraints on the atmospheric gas abundances and cloud properties in widely separated giant exoplanets with one observation of the reflected light, without the degeneracy between the gas abundance and the cloud pressure as discussed 
above. Not knowing the planetary radius does not substantially affect this capability. To our knowledge, this is the first time such capability is demonstrated with realistic uncertainty estimates and Bayesian retrievals. Also, as we have shown in Damiano \& $\mathrm{Hu}$ (2020), the abundance of the cloud-forming gas (e.g., $\mathrm{H}_{2} \mathrm{O}$ in $47 \mathrm{UMa}$ b) below the cloud can be constrained from the reflected light spectra.

Finally, EXOREL ${ }^{\Re \mathfrak{R}}$ has been designed to be applicable to the population of cold gaseous planets orbiting at the semimajor axis of $>1$ and $<10$ au from solar-like stars. These planets may be described with a single or double layers of clouds, made of water and/or ammonia ices depending on temperature and optical depth. 47 Uma b, the target considered in this work, is one of the planets in this category. The degenerate solutions and the strategies of mitigation presented here are thus broadly applicable to the planets in the aforementioned category.

In conclusion, we present simulations of starshade-enabled reflected light spectroscopy of widely separated giant exoplanets and use Bayesian retrievals to assess strategies to improve the constraints on the atmospheric properties. We show that a degenerate scenario with a lower gas abundance and a deeper or absent cloud than the truth can be retrieved when the observation is limited to a $\sim 20 \%$ band in $600-800 \mathrm{~nm}$ and has an $\mathrm{S} / \mathrm{N}$ of $\sim 18$. Widening the wavelength coverage to include $800-1000 \mathrm{~nm}$ is the most effective way to eliminate the degenerate solution. Doubling the integration time or repeating the observation at a different, less-than-optimal orbital phase would also eliminate the degenerate solution. However, observation overheads must be taken into consideration as the mission resources are limited. Doubling the integration time likely comes with very little overhead, but costs twice as much station-keeping fuel for the starshade. Moreover, the starshade would need to be moved closer to the telescope to repeat the observation in the red band. The multiphase option requires doubling overheads and the fuel associated with slewing the starshade back to the same target. Although these results are based on starshade simulations, they are generally applicable to high-contrast imaging and spectroscopy with a coronagraph with similar wavelength coverage, spectral resolution, and $\mathrm{S} / \mathrm{N}$. The work presented here will be important to design and define the wavelength bandwidth and the observation plan of exoplanet direct-imaging experiments in the future.

The authors thank Dr. Graça M. Rocha for helpful discussions in the preparation of this manuscript, Dr. Stefan Martin for providing the optical throughput and the detector quantum efficiency used by Roman Coronagraph and HabEx, and Dr. Andrew Romero-Wolf for providing Roman's QE value at EOL. Finally, we also thank the anonymous referee for the valuable comments provided which greatly helped to improve the manuscript. This work was supported in part by the NASA WFIRST Science Investigation Teams grant \#NNN16D016T. This research was carried out at the Jet Propulsion Laboratory, California Institute of Technology, under a contract with the National Aeronautics and Space Administration.

\section{ORCID iDs}

Mario Damiano (1) https://orcid.org/0000-0002-1830-8260

Renyu Hu (i) https://orcid.org/0000-0003-2215-8485

Sergi R. Hildebrandt (1) https://orcid.org/0000-00030220-0009

\section{References}

Akeson, R., Armus, L., Bachelet, E., et al. 2019, arXiv:1902.05569 Burrows, A., Sudarsky, D., \& Hubeny, I. 2004, ApJ, 609, 407 Cahoy, K. L., Marley, M. S., \& Fortney, J. J. 2010, ApJ, 724, 189 Carrión-González, Ó, Muñoz, A. G., Cabrera, J., et al. 2020, A\&A, 640, A136 Damiano, M., \& Hu, R. 2020, AJ, 159, 175

Ertel, S., Defrère, D., Hinz, P., et al. 2020, AJ, 159, 177

Gaudi, B. S., Seager, S., Mennesson, B., et al. 2020, arXiv:2001.06683

Hilgemann, E., Shaklan, S., McKeithen, D., et al. 2019, NASA Starshade Technology Development Activity Milestone Report, 3, https://exoplanets. nasa.gov/internal_resources/1544/

Hu, R. 2014, arXiv: 1412.7582

Hu, R. 2019, ApJ, 887, 166

Janson, M., Bergfors, C., Goto, M., Brandner, W., \& Lafrenière, D. 2010, ApJL, 710, L35

Konopacky, Q. M., Barman, T. S., Macintosh, B. A., \& Marois, C. 2013, Sci, 339, 1398

Lupu, R. E., Marley, M. S., Lewis, N., et al. 2016, AJ, 152, 217

MacDonald, R. J., Marley, M. S., Fortney, J. J., \& Lewis, N. K. 2018, ApJ, 858,69

Macintosh, B., Graham, J. R., Barman, T., et al. 2015, Sci, 350, 64

Madhusudhan, N., \& Burrows, A. 2012, ApJ, 747, 25

Nayak, M., Lupu, R., Marley, M. S., et al. 2017, PASP, 129, 034401

Nemati, B., Krist, J. E., \& Mennesson, B. 2017, Proc. SPIE, 10400, 1040007

Rajan, A., Rameau, J., De Rosa, R. J., et al. 2017, AJ, 154, 10

Robinson, T. D., Stapelfeldt, K. R., \& Marley, M. S. 2016, PASP, 128, 025003

Samland, M., Mollière, P., Bonnefoy, M., et al. 2017, A\&A, 603, A57

Sato, M., \& Hansen, J. E. 1979, JAtS, 36, 1133

Seager, S., Kasdin, N. J., Booth, J., et al. 2019, BAAS, 51, 106

Spergel, D., Gehrels, N., Baltay, C., et al. 2015, arXiv:1503.03757

Sudarsky, D., Burrows, A., \& Hubeny, I. 2003, ApJ, 588, 1121

Sudarsky, D., Burrows, A., \& Pinto, P. 2000, ApJ, 538, 885

Wagner, K., Apai, D., Kasper, M., et al. 2016, Sci, 353, 673 